\title{
Novel Pattern of Iron Deposition in the Fascicula Nigrale in Patients with Parkinson's Disease: A Pilot Study
}

\author{
Miriam E. Peckham, ${ }^{1}$ Khashayar Dashtipour, ${ }^{2}$ Barbara A. Holshouser, ${ }^{1}$ Camellia Kani, ${ }^{2}$ \\ Alex Boscanin, ${ }^{1}$ Kayvan Kani, ${ }^{1}$ and Sheri L. Harder ${ }^{1}$ \\ ${ }^{1}$ Department of Radiology, School of Medicine, Loma Linda University, 11234 Anderson Street, Loma Linda, CA 92354, USA \\ ${ }^{2}$ Department of Neurology, School of Medicine, Loma Linda University, 11234 Anderson Street, Loma Linda, CA 92354, USA
}

Correspondence should be addressed to Khashayar Dashtipour; kdashtipour@llu.edu

Received 7 March 2016; Revised 31 May 2016; Accepted 31 May 2016

Academic Editor: Paul Sijens

Copyright ( 2016 Miriam E. Peckham et al. This is an open access article distributed under the Creative Commons Attribution License, which permits unrestricted use, distribution, and reproduction in any medium, provided the original work is properly cited.

\begin{abstract}
Background and Purpose. To determine whether the pattern of iron deposition in the fascicula nigrale in patients with Parkinson's disease would be different from age-matched controls by utilizing quantitative susceptibility mapping to measure susceptibility change. Methods. MRIs of the brain were obtained from 34 subjects, 18 with Parkinson's disease and 16 age- and gender-matched controls. Regions of interest were drawn around the fascicula nigrale and substantia nigra using SWI mapping software by blinded investigators. Statistical analyses were performed to determine susceptibility patterns of both of these regions. Results. Measurements showed significantly increased susceptibility in the substantia nigra in Parkinson's patients and an increased rostralcaudal deposition of iron in the fascicula nigrale in all subjects. This trend was exaggerated with significant correlation noted with increasing age in the Parkinson group. Conclusion. The pattern of an exaggerated iron deposition gradient of the fascicula nigrale in the Parkinson group could represent underlying tract dysfunction. Significant correlation of increasing iron deposition with increasing age may be a cumulative effect, possibly related to disease duration.
\end{abstract}

\section{Introduction}

Parkinson's disease (PD) is a neurodegenerative disorder which has been found by multiple investigators to be associated with cell loss in the striatonigral dopaminergic pathway as well as the substantia nigra (SN) $[1,2]$. Anatomically, the striatonigral tract and nigrostriatal tract make up the fascicula nigrale (FN) which was first described by Harder et al. as a mineralized structure extending from the globus pallidus (GP) to the substantia nigra (SN) (Figure 1) [3]. This tract has been hypothesized to take part in iron transport between the basal nuclei and midbrain $[3,4]$. On susceptibility weighted imaging (SWI) this tract is identified as a linear focus of increased susceptibility extending from the medial aspect of the GP rostrally to the anterior aspect of the substantia nigra caudally (Figures 2 and 3).

The SN has been found to have increased accumulation of iron in PD by multiple investigators; because of this, the mechanism of iron homeostasis in this disease state has been a subject of interest $[5,6]$. Transferrin is the brain's primary iron-transport protein and was found by Lee and Andersen to have increased levels within dopaminergic neurons of the SN in studies on rat and monkey models of PD [7]. The movement of transferrin to the storage sites has been hypothesized by Olanow and Youdim to be through axonal transport and has been studied in rats where transferrin has been tracked from the retina into the optic nerve, chiasm, and eventually the super colliculus by Moos et al. $[8,9]$. Lactoferrin and DMT1 (divalent metal transporter 1), additional transporters of reactive iron, have also been found by Hirsch to be overexpressed within dopaminergic neurons of SN cells in mouse models of PD $[10,11]$. Hirsch also found increased levels of DMT1 in the brain to be correlated with increasing age corresponding to known age related cellular iron accumulation as described by Hallgren and Sourander [10-12]. An increase of these transport proteins within the SN may partly account for disrupted iron homeostasis $[7,10,11]$. 


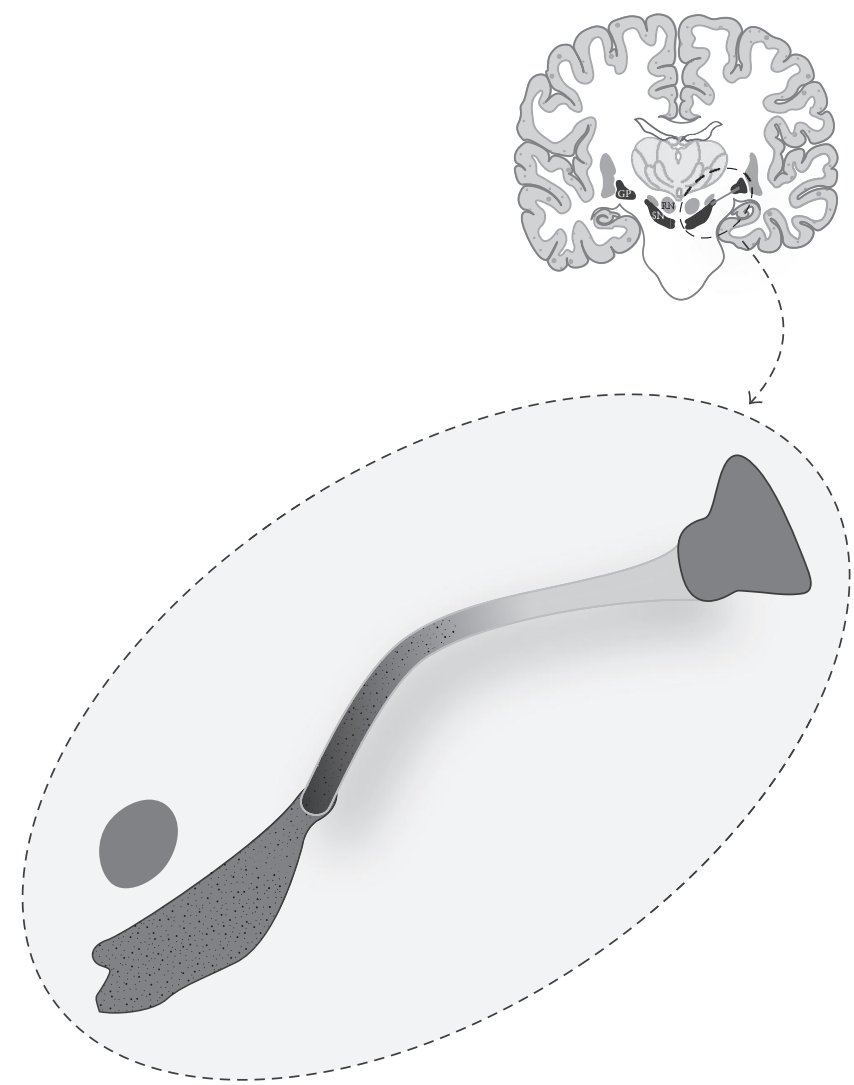

FIGURE 1: Illustration of the fascicula nigrale, a mineralized midbrain structure extending from the GP to the SN.

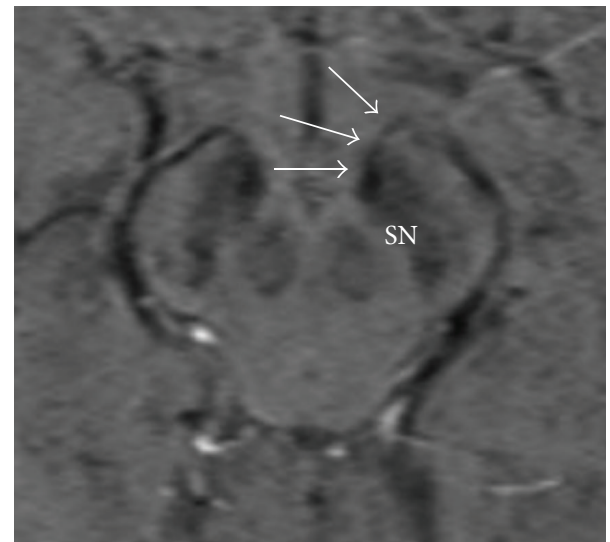

FIGURE 2: The FN (delineated by white arrows) is shown at its junction with the SN (SWI, $2 \mathrm{~mm}$ ).

Hallgren and Sourander first characterized midbrain mineralization patterns in postmortem subjects in 1958 [12]. Multiple investigators have developed techniques to measure iron deposition noninvasively using high-field strength spinecho T2 weighted MRI sequences such as relaxometry (R2*), quantitative susceptibility mapping, and diffusion tensor imaging [5, 13-16]. SWI was first developed in the mid1990 s using phase as a means to enhance contrast in T2* sequences. Quantitative susceptibility mapping grew out of the SWI precursor as a method to use phase information to measure iron content in the midbrain, as discussed by Haacke et al. [15]. Mapping of SWI phase shift values was positively correlated to Hallgren and Sourander's original autopsy findings in in vivo healthy controls by Zhang et al. validating the application of SWI mapping as a noninvasive technique for iron measurement [16]. Quantitative susceptibility mapping was also found to exhibit superb contrast of brain structures and substructures, some of which were not evident on $\mathrm{R} 2 *$, magnitude, or frequency images by Deistung et al. [14]. Multiple studies using noninvasive SWI and R2* have been performed, replicating previous postmortem findings of increased iron deposition in the brain of patients with PD [2, 6, 17-24]. Quantitative susceptibility mapping was originally found to be more sensitive than $\mathrm{R} 2 *$ in detecting iron deposition changes in patients with multiple sclerosis by Haacke et al. Murakami et al. and Barbosa et al. compared these techniques in PD brains, and quantitative susceptibility mapping was found to be more sensitive with highly accurate discrimination of patient and control groups $[17,25,26]$. As quantitative susceptibility mapping is currently the most accurate and sensitive measurement technique for midbrain iron deposition, it was used in this study to quantify iron deposition in the $\mathrm{SN}$ and FN.

It has been hypothesized by Graham et al. that increased susceptibility in the $\mathrm{SN}$ is due to accumulation of iron free radicals [5]. Iron deposition patterns in the FN have never been previously measured. Because of its involvement in 

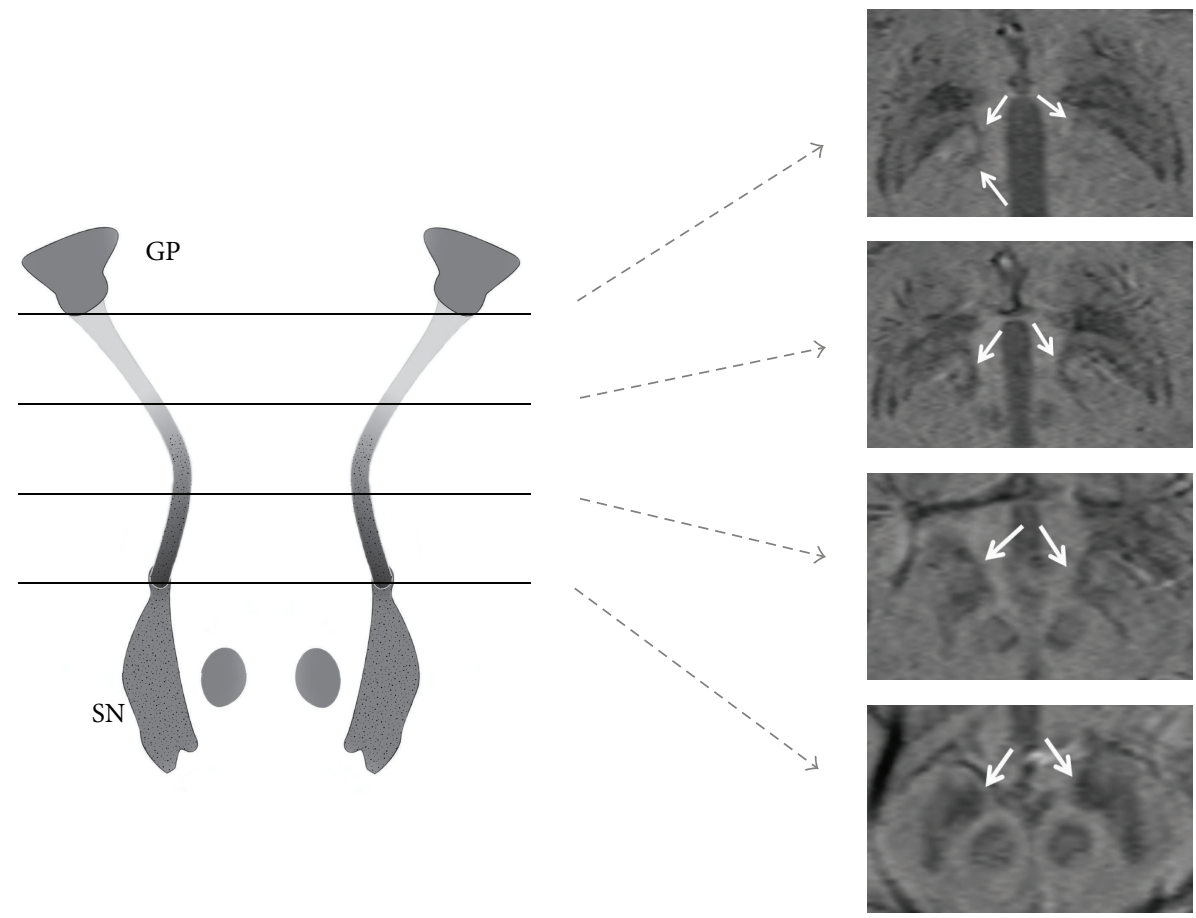

FIGURE 3: Illustration depicting the appearance of multiple slices through the FN (white arrows on SWI images, 2 mm thk) shown at its rostral junction with the globus pallidus descending to its caudal junction with the substantia nigra.

the striatonigral dopaminergic pathway and its hypothesized role in iron transport, further investigation of this tract for pathologic iron deposition is warranted. Our group recently demonstrated the heterogeneity of iron accumulation in the brain, among patients with PD, using SWI [6]. The purpose of this study was to use susceptibility mapping to measure the pattern of iron deposition in the fascicula nigrale in PD patients. It was hypothesized that PD patients would have increased iron deposition in the both the FN and the SN.

\section{Methods}

2.1. Subjects. Eighteen patients (11 males and 7 females, mean age 69.1 years, and range: 52-82 years) with a new diagnosis of idiopathic PD were recruited by a movement disorders specialist from Loma Linda University Medical Center (LLUMC) Movement Disorder Clinic Department of Neurology, based on the UK Parkinson's Disease Society Brain Bank's criteria (Table 1). All subjects with PD had a brain MRI performed within 3 months of initial diagnoses. A PD treatment regimen was not yet implemented at the time of MRI. Patients with atypical parkinsonism, concomitant vascular parkinsonism, or parkinsonism due to neuroleptics were excluded from the study. Sixteen non-PD subjects (5 males and 11 females) with mean age of 64.4 years (range: 53-90 years) were recruited from LLUMC Neurology Clinic after ruling out PD. Subjects with past or current history of cognitive impairment, stroke, head trauma, brain lesions, or other neurodegenerative and neuropsychiatric diseases were excluded. Our Institutional Review Board approved the study. Informed consent was obtained from all study participants.
TABLE 1: Demographic and clinical characteristics of the study groups.

\begin{tabular}{lccc}
\hline & PD & Control & $P$ value \\
& $N=18$ & $N=16$ & \\
\hline M:F & $11: 7$ & $5: 11$ & 0.081 \\
Mean age (years) & $69.1 \pm 11.2^{*}$ & $64.4 \pm 6.1$ & 0.138 \\
Time of diagnosis to MRI & $1.1 \pm 0.3$ & N/A & \\
(months) & $15: 3$ & $16: 0$ & \\
Handedness right:left & &
\end{tabular}

Data are shown as mean \pm SD.

${ }^{*}$ Because the diagnosis was de novo, this also represents mean age of $\mathrm{PD}$ onset.

2.2. Magnetic Resonance Imaging and Image Processing. Conventional MR imaging and SWI were performed with a $3 \mathrm{~T}$ whole body MR scanner (Tim/Trio, Siemens Medical Solutions, Erlangen, Germany) using a 12-channel head array coil. The following sequences were acquired on all subjects: sagittal T1 weighted 3D MPRage (TR/TE/TI = 1950/226/900 msec, $1 \times 1 \times 1 \mathrm{~mm}$ voxel size), sagittal 3D T2 weighted SPACE, (TR/TE3200/458 msec, $1 \times 1 \times 1 \mathrm{~mm}$ voxel size), axial fat-saturated FLAIR (TR/TE/TI $=9000 / 77 / 2500 \mathrm{msec}$, $3 \mathrm{~mm}$ thick, $.9 \mathrm{~mm}$ spacing), axial diffusion (EPI single shot $\mathrm{TR} / \mathrm{TE}=5700 / 103 \mathrm{msec}, 1.5 \times 1.5 \times 3 \mathrm{~mm}$ voxel size $.9 \mathrm{~mm}$ spacing), and axial 3D SWI (TR/TE $=29 / 20 \mathrm{msec}, 1.0 \times 0.5 \times$ $2.0 \mathrm{~mm}$ voxel size). T1 and T2 weighted sequences were used to aid in anatomic localization.

2.3. Susceptibility Mapping and Region of Interest Data Analysis. SWI mapping data were calculated with SPIN (Signal 


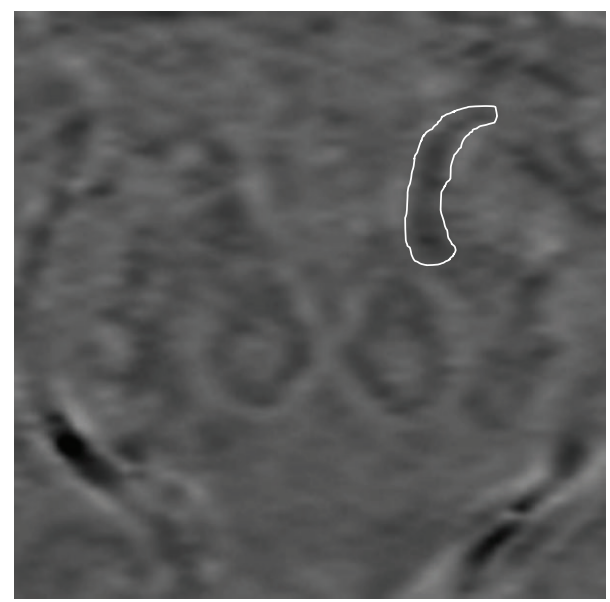

FIGURE 4: ROI around the FN as seen on mIPs of susceptibility maps (SWIM, 8 thk).

Processing In Nuclear magnetic resonance, developed by the MRI Institute for Biomedical Research, Detroit, Michigan) $[27,28]$. Susceptibility maps were generated from the vertex to the medulla using magnitude and phase SWI images. The images were prefiltered by the manufacturer's software and no additional filter was applied. An inverse filter threshold of 0.1 , iterative SWI mapping vein threshold of $200, k$-space threshold of 0.1 , and iterations times of 3 were applied in generating the susceptibility map. Minimum intensity maps (mIPs) were created from four slices that were each $2 \mathrm{~mm}$ thick. Two blinded, independent readers drew regions of interest (ROI) around the bilateral FN and SN on these mIP images for each subject. These ROIs were determined by tracing along the outside of the mineralized portion of the substantia nigra. ROI tracings were also drawn just outside of the mineralized borders of the putamen, thalami, red nuclei, dentate nuclei, and precentral gyrus (at the omega sign) at their most prominent slice bilaterally. A threshold of 1000 was applied to each ROI, obtained by averaging the subcortical white matter in all subjects (AVG 1000.06 \pm 0.5 ). Maximum intensity values in each ROI above this threshold were recorded. The susceptibility intensity values were taken from the region of interest drawn around the SN at its most prominent slice bilaterally. The FN was measured at all slices it was visualized on from its superior connection to the globus pallidus to its inferior connection with the SN (Figure 4). The number of $8 \mathrm{~mm}$ slices the tract was present on ranged between 3 and 10, averaging approximately 5.0 slices $(40 \mathrm{~mm})$ on the right and 5.1 slices $(41 \mathrm{~mm})$ on the left. The FN was divided into two parts, rostral and caudal. If there were an odd number of slice measurements, then favor was given to the caudal half. In the case of a measurement agreement falling outside of the $95 \%$ confidence interval, a third reader made the final determination. A third reader had to be utilized for 4 of 68 tract measurements.

2.4. Statistical Analysis. All statistics analyses were performed using SPSS statistical software (version 13.0, SPSS Inc., Chicago, Illinois). $P$ values less than 0.05 were considered statistically significant. Parametric tests were used to determine significant difference between the two cohorts and nonparametric tests (Mann-Whitney $U$ test) were used if normality assumptions could not be met. Spearman correlation analysis was conducted to analyze the relationship between the indicators. Age and gender were modeled as covariates to correct for age- or gender-related iron deposition in the binary logistic regression analysis. To verify the reproducibility of the findings (intrarater variability) and to determine interrater reliability, intraclass correlation coefficients (ICC) were calculated.

\section{Results}

Susceptibility intensity values for the FN were redefined by the same rater to assess the reproducibility of the results. The correlation was 0.955 comparing the rostral to caudal deltas of the FN, which is an acceptable level of reproducibility. Susceptibility intensity values of the FN were also compared between two readers showing correlation of 0.733 when comparing the rostral to caudal deltas, which is an acceptable level of reproducibility. Finally, susceptibility values of the SN were compared between two readers showing correlation of 0.871 , which is an acceptable level of reproducibility.

There was significantly increased susceptibility of the SN in the PD group $(P=0.012)$.

To assess MRI detectable iron levels in the whole FN structure, the mean and standard deviation (SD) of iron deposition along the entire tract were calculated by averaging the maximum values obtained in each ROI. These results showed a trend of increased iron deposition (1083.78 versus 1069.95 in ppm of iron, $P=0.065)$ and wider standard deviation in the PD group (37.00 versus 25.77, $P=0.169$ ).

All subjects were found to have a descending pattern of increasing iron deposition along the FN with higher susceptibility levels in the caudal aspect of the tract (Figure 5). The iron deposition at the caudal aspect of the tract showed a nonsignificant trend of increased susceptibility in the PD group compared to controls $(1105.13 \pm 45.8$ versus $1083.00 \pm$ 13.8 in ppm of iron, $P=0.073$ ) (Figure 6). An illustrated summary of results is provided in Figure 7 , representing control and PD groups, respectively.

The difference between the lowest value in the rostral and highest value in the caudal aspects of the tract (designated as delta) was calculated and showed a nearly significant trend of an increased delta in the PD group $(99.9 \pm 82.9$ versus $57.8 \pm$ 29.1, $P=0.056)$.

Age in the PD group was positively correlated with the mean $(r=0.632 ; P=0.005)$ and the standard deviation of iron deposition in the FN $(r=0.530 ; P=0.024)$ and deposition at the caudal aspect of the FN $(r=0.575$; $P=0.013)$, as well as an increased delta $(r=0.626, P=$ $0.005)$. There was no significant correlation in the control group between age and iron deposition. Age and gender were modeled as covariates to correct for age- and sex-related iron deposition in the binary logistic regression analysis. The iron deposition at the caudal aspect of the tract, the mean, and delta was all insignificant after adjustment for age and sex. 


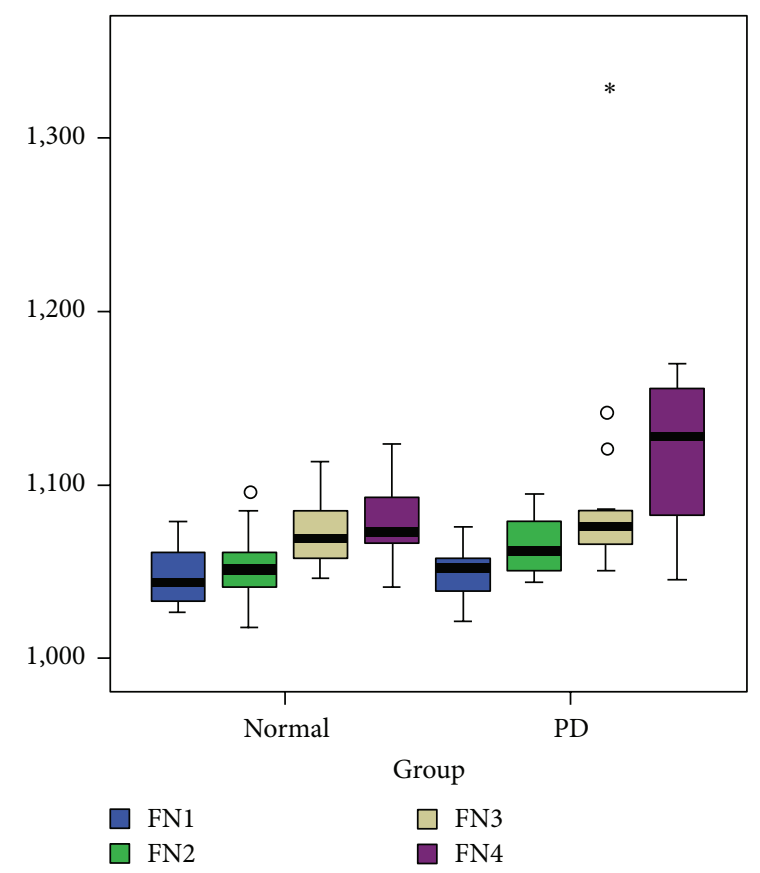

FIGURE 5: Descending pattern of increasing iron deposition along the FN (FN1 is rostral and FN4 is caudal and also contains the averages of slices more caudal to FN4 when present). * = outlying value.

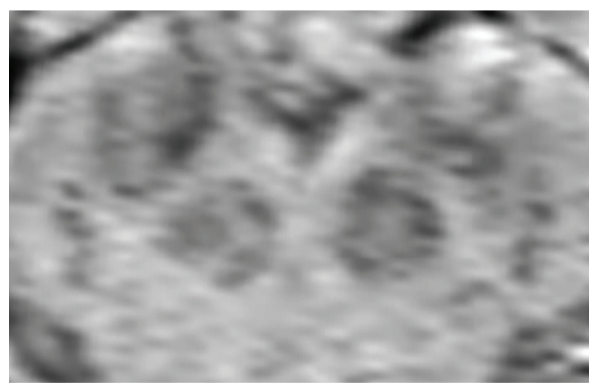

(a)

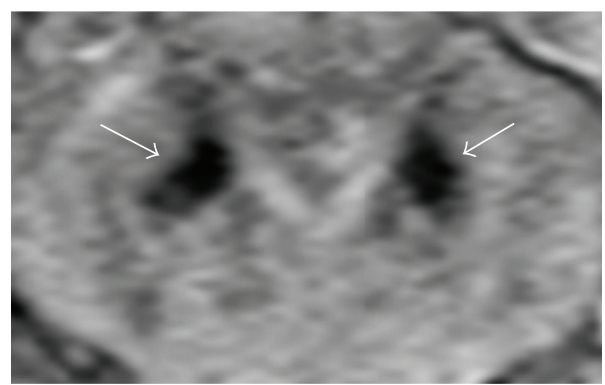

(b)

FIGURE 6: Axial images through the level of the substantia nigra demonstrating iron deposition patterns in a control subject (a) and PD subject (b), with the PD subject showing focally high susceptibility at the caudal aspect of the FN tract (white arrows) (SWI, $2 \mathrm{~mm}$ ).

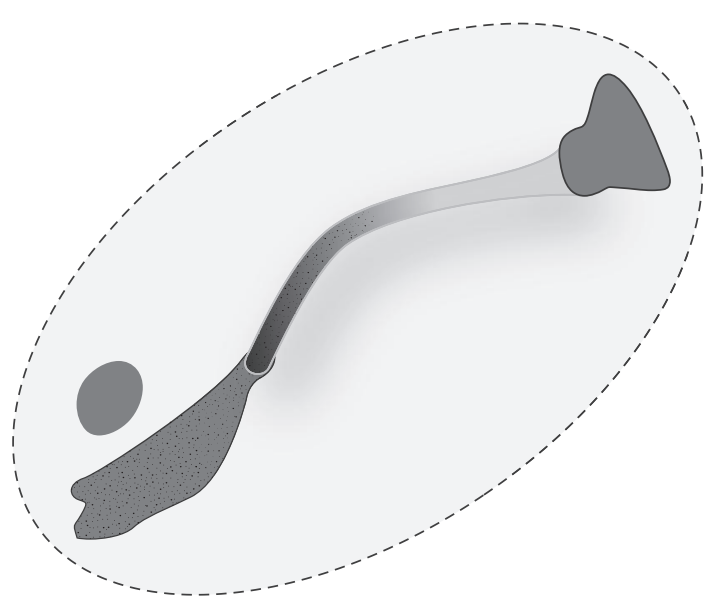

Control

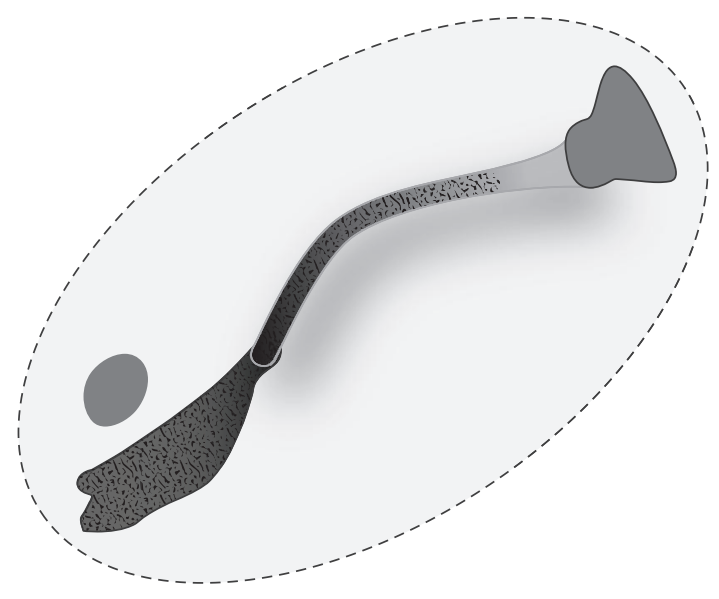

PD

FIGURE 7: Illustrated trends of iron deposition in the FN and SN in control and PD groups. 
All other measured regions of interest including the gray matter, putamen, thalami, red nuclei, and dentate nuclei showed no significant difference in iron deposition between $\mathrm{PD}$ and control groups.

\section{Discussion}

There is significantly increased iron deposition in the SN in the PD group, in line with multiple prior susceptibility studies $[2,5,16,17,19-24]$. Our results demonstrate a novel trend of exaggerated iron deposition, increasing from the rostral to caudal FN, in the PD group, with significant correlation with increased age. The findings of increased iron deposition in the FN with PD patient age and trend of an exaggerated gradient may suggest an underlying pathologic process.

The correlation of these findings with increased patient age is an interesting finding of uncertain etiology. This may be related to disease prolongation and severity, possibly while the disease was subclinical as these patients were all at early stage.

Limitations in this study include white matter contamination, which was potentially more exaggerated in the FN because of the small region of interest. Because of this maximum values were studied to decrease the effect of white matter dilution. As this was a pilot study, the number of subjects to achieve adequate power had never been previously established for reference. Some values reaching only nearsignificance could suggest that the overall subject size may have been too small to detect a difference between groups. Conversely, normal variability in deposition patterns across age groups as previously described by Harder et al. may have been exaggerated by the small sampling size [3]. Further studies would benefit from increased power to establish whether these deposition trends are truly abnormal in the PD group. A further limitation of the study was the uneven sex distribution between control and PD groups. To correct this discrepancy, gender and age were modeled as covariates in the binary logistic regression analysis. Another study limitation was using prefiltered data for measurement. This likely decreased the sensitivity of susceptibility measurements, which may have diluted the noted trends. A nonfiltered set of normal values would be helpful in developing a conversion factor for the filtered data.

\section{Conclusion}

PD has been associated with neuronal cell loss in the striatonigral dopaminergic pathway. The novel pattern of an exaggerated iron deposition gradient of the FN in PD could represent underlying tract dysfunction. Significant correlation of increasing iron deposition with increasing age may be a cumulative effect, related to disease duration.
Abbreviations
PD: Parkinson's disease
SN: Substantia nigra
FN: Fascicula nigrale
GP: Globus pallidus.

\section{Competing Interests}

The authors declare that there are no competing interests regarding the publication of this paper.

\section{Acknowledgments}

Thanks are due to Mr. Sean Olson for producing all illustrations.

\section{References}

[1] J. K. Dreyer, "Three mechanisms by which striatal denervation causes breakdown of dopamine signaling," The Journal of Neuroscience, vol. 34, no. 37, pp. 12444-12456, 2014.

[2] A. K. Lotfipour, S. Wharton, S. T. Schwarz et al., "High resolution magnetic susceptibility mapping of the substantia nigra in Parkinson's disease," Journal of Magnetic Resonance Imaging, vol. 35, no. 1, pp. 48-55, 2012.

[3] S. L. Harder, K. M. Hopp, H. Ward, H. Neglio, J. Gitlin, and D. Kido, "Mineralization of the deep gray matter with age: a retrospective review with susceptibility-weighted MR imaging," American Journal of Neuroradiology, vol. 29, no. 1, pp. 176-183, 2008.

[4] E. S. Manova, C. A. Habib, A. S. Boikov et al., "Characterizing the mesencephalon using susceptibility-weighted imaging," American Journal of Neuroradiology, vol. 30, no. 3, pp. 569-574, 2009.

[5] J. M. Graham, M. N. J. Paley, R. A. Grünewald, N. Hoggard, and P. D. Griffiths, "Brain iron deposition in Parkinson's disease imaged using the PRIME magnetic resonance sequence," Brain, vol. 123, part 12, pp. 2423-2431, 2000.

[6] K. Dashtipour, M. Liu, C. Kani et al., "Iron accumulation is not homogenous among patients with Parkinson's disease," Parkinson's Disease, vol. 2015, Article ID 324843, 8 pages, 2015.

[7] D. W. Lee and J. K. Andersen, "Iron elevations in the aging Parkinsonian brain: a consequence of impaired iron homeostasis?" Journal of Neurochemistry, vol. 112, no. 2, pp. 332-339, 2010.

[8] C. W. Olanow and M. B. H. Youdim, "Iron and neurodegeneration: prospects for neuroprotection," in Neurodegeneration and Neuroprotection in Parkinson's Disease, chapter 4, pp. 5567, Academic Press, London, UK, 1996.

[9] T. Moos, N. Bernth, Y. Courtois, and E. H. Morgan, "Developmental iron uptake and axonal transport in the retina of the rat," Molecular and Cellular Neuroscience, vol. 46, no. 3, pp. 607-613, 2011.

[10] E. C. Hirsch, "Altered regulation of iron transport and storage in Parkinson's disease," Journal of neural transmission. Supplementum, no. 71, pp. 201-204, 2006.

[11] E. C. Hirsch, "Iron transport in Parkinson's disease," Parkinsonism and Related Disorders, vol. 15, supplement 3, pp. S209-S211, 2009.

[12] B. Hallgren and P. Sourander, "The effect of age on the nonhaemin iron in the human brain," Journal of Neurochemistry, vol. 3, no. 1, pp. 41-51, 1958.

[13] P. Péran, A. Cherubini, F. Assogna et al., "Magnetic resonance imaging markers of Parkinson's disease nigrostriatal signature," Brain, vol. 133, no. 11, pp. 3423-3433, 2010.

[14] A. Deistung, A. Schäfer, F. Schweser, U. Biedermann, R. Turner, and J. R. Reichenbach, "Toward in vivo histology: a comparison of quantitative susceptibility mapping (QSM) with magnitude-, 
phase-, and R2 ${ }^{*}$-imaging at ultra-high magnetic field strength," NeuroImage, vol. 65, pp. 299-314, 2013.

[15] E. M. Haacke, S. Liu, S. Buch, W. Zheng, D. Wu, and Y. Ye, "Quantitative susceptibility mapping: current status and future directions," Magnetic Resonance Imaging, vol. 33, no. 1, pp. 1-25, 2015.

[16] J. Zhang, Y. Zhang, J. Wang et al., "Characterizing iron deposition in Parkinson's disease using susceptibility-weighted imaging: an in vivo MR study," Brain Research, vol. 1330, pp. 124-130, 2010.

[17] Y. Murakami, S. Kakeda, K. Watanabe et al., "Usefulness of quantitative susceptibility mapping for the diagnosis of Parkinson disease," American Journal of Neuroradiology, vol. 36, no. 6, pp. 1102-1108, 2015.

[18] M. Wieler, M. Gee, and W. R. W. Martin, "Longitudinal midbrain changes in early Parkinson's disease: iron content estimated from $R_{2}^{*}$ /MRI," Parkinsonism and Related Disorders, vol. 21, no. 3, pp. 179-183, 2015.

[19] F. J. A. Meijer and B. Goraj, "Brain MRI in Parkinson's disease," Frontiers in Bioscience, vol. 6, no. 2, pp. 360-369, 2014.

[20] P. Riederer, A. Dirr, M. Goetz, E. Sofic, K. Jellinger, and M. B. H. Youdim, "Distribution of iron in different brain regions and subcellular compartments in Parkinson's disease," Annals of Neurology, vol. 32, supplement 1, pp. S101-S104, 1992.

[21] P. D. Griffiths, B. R. Dobson, G. R. Jones, and D. T. Clarke, "Iron in the basal ganglia in Parkinson's disease. An in vitro study using extended X-ray absorption fine structure and cryoelectron microscopy," Brain, vol. 122, no. 4, pp. 667-673, 1999.

[22] D. T. Dexter, A. Carayon, F. Javoy-Agid et al., "Alterations in the levels of iron, ferritin and other trace metals in Parkinson's disease and other neurodegenerative diseases affecting the basal ganglia," Brain, vol. 114, part 4, pp. 1953-1975, 1991.

[23] Y. Wang, S. R. Butros, X. Shuai et al., "Different iron-deposition patterns of multiple system atrophy with predominant parkinsonism and idiopathetic Parkinson diseases demonstrated by phase-corrected susceptibility-weighted imaging," American Journal of Neuroradiology, vol. 33, no. 2, pp. 266-273, 2012.

[24] S. Haller, S. Badoud, D. Nguyen et al., "Differentiation between Parkinson disease and other forms of Parkinsonism using support vector machine analysis of susceptibility-weighted imaging (SWI): initial results," European Radiology, vol. 23, no. 1, pp. 1219, 2013.

[25] J. H. O. Barbosa, A. C. Santos, V. Tumas et al., "Quantifying brain iron deposition in patients with Parkinson's disease using quantitative susceptibility mapping, R2 and R2*," Magnetic Resonance Imaging, vol. 33, no. 5, pp. 559-565, 2015.

[26] E. M. Haacke, M. Makki, Y. Ge et al., "Characterizing iron deposition in multiple sclerosis lesions using susceptibility weighted imaging," Journal of Magnetic Resonance Imaging, vol. 29, no. 3, pp. 537-544, 2009.

[27] E. M. Haacke, J. Tang, J. Neelavalli, and Y. C. Cheng, "Susceptibility mapping as a means to visualize veins and quantify oxygen saturation," Journal of Magnetic Resonance Imaging, vol. 32, pp. 663-676, 2010.

[28] J. Tang, S. Liu, J. Neelavalli, Y. C. N. Cheng, S. Buch, and E. M. Haacke, "Improving susceptibility mapping using a threshold-based K-space/image domain iterative reconstruction approach," Magnetic Resonance in Medicine, vol. 69, no. 5, pp. 1396-1407, 2013. 


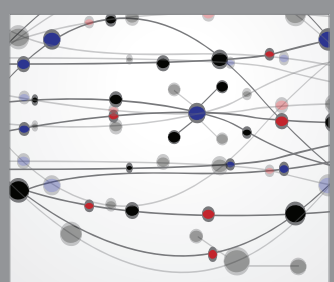

The Scientific World Journal
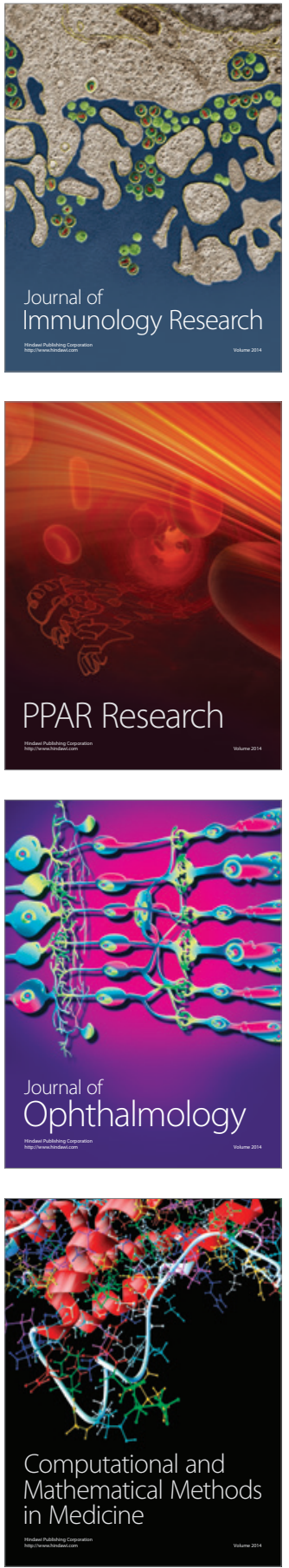

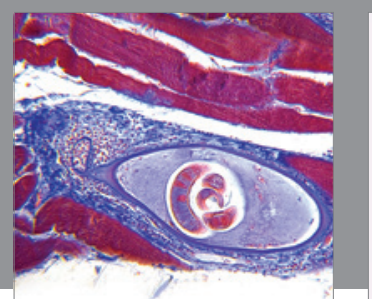

Gastroenterology Research and Practice

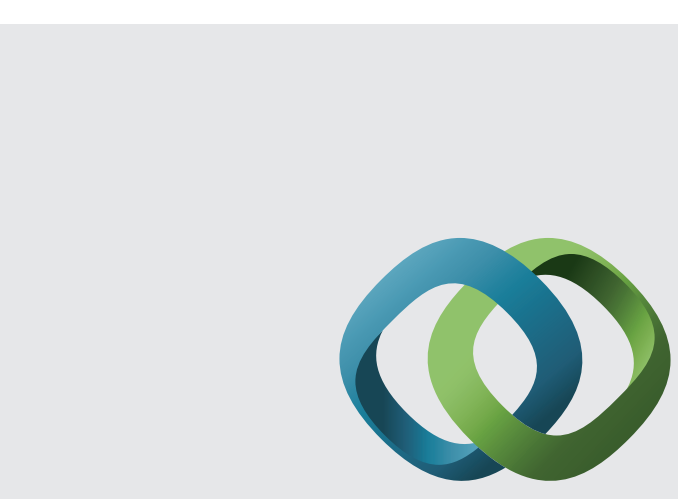

\section{Hindawi}

Submit your manuscripts at

http://www.hindawi.com
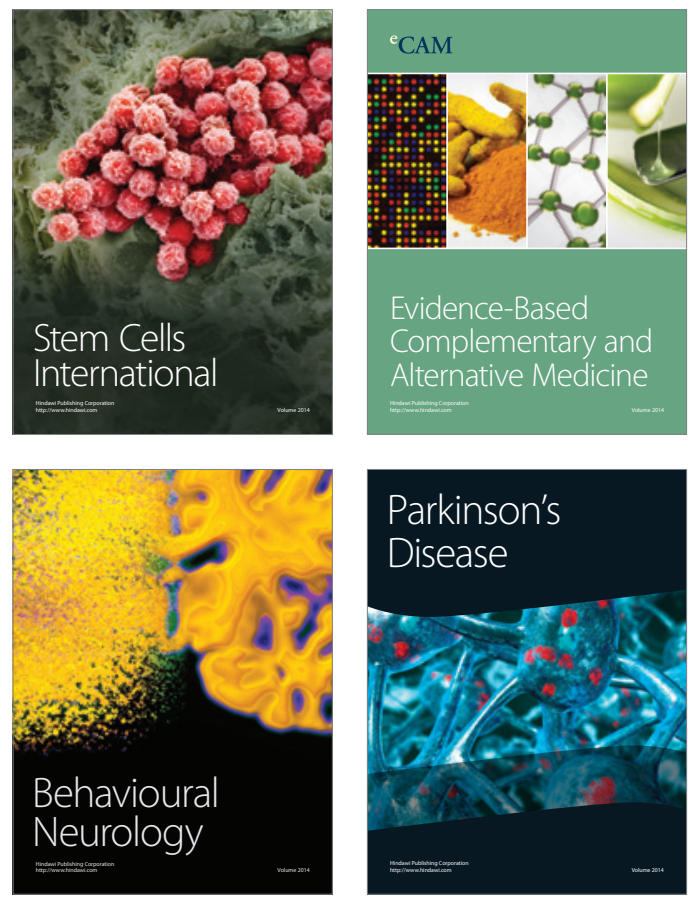
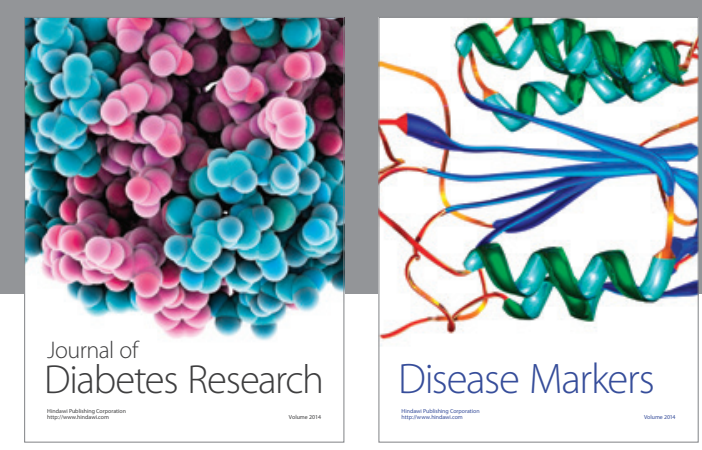

Disease Markers
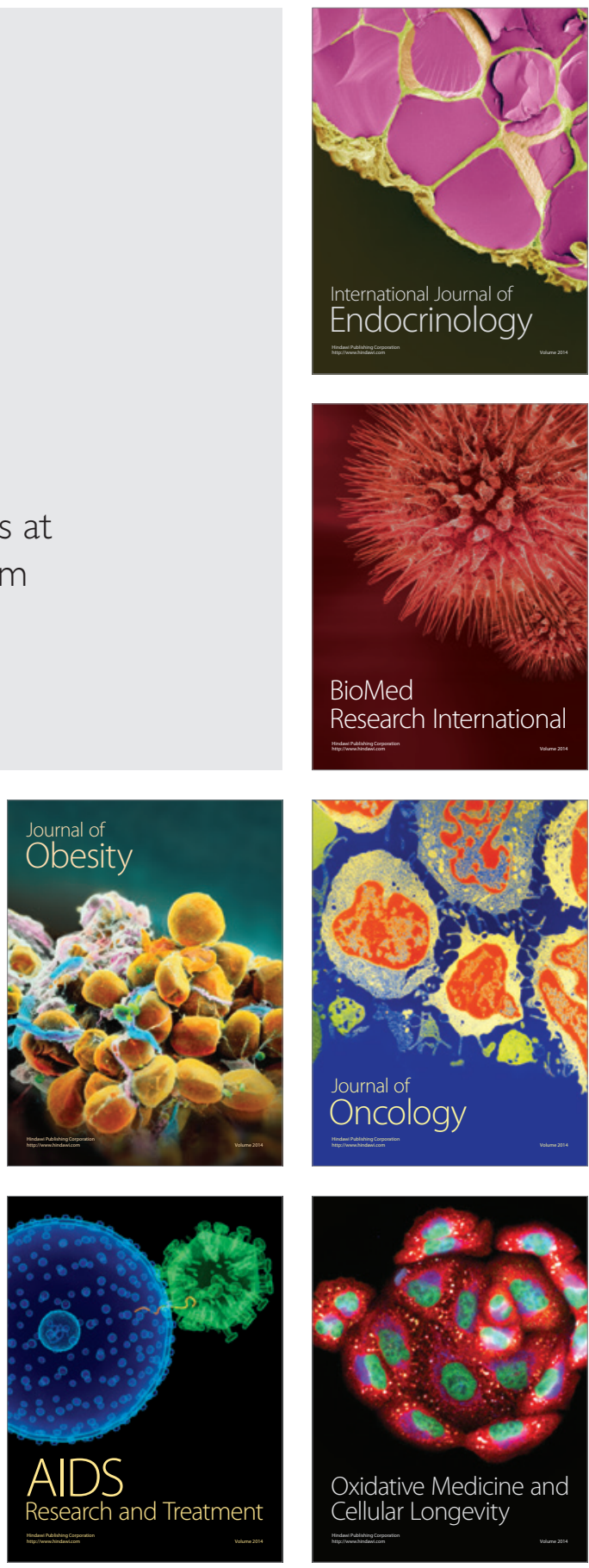